\title{
From Microscopy Images to Models of Cellular Processes
}

\author{
Yoav Freund \\ Computer Science and Engineering \\ University of California, San Diego, USA
}

The advance of fluorescent tagging and of confocal microscopy is allowing biologists to image biochemical processes at a level of detail that was unimaginable just a few years ago. However, as the analysis of these images is done mostly by hand, there is a severe bottleneck in transforming these images into useful quantitative data that can be used to evaluate mathematical models.

One of the inherent challenges involved in automating this transformation is that image data is highly variable. This requires a recalibration of the image processing algorithms for each experiment. We use machine learning methods to enable the experimentalist to calibrate the image processing methods without having any knowledge of how these methods work. This, we believe, will allow the rapid integration of computer vision methods with confocal microscopy and open the way to the development of quantitative spatial models of cellular processes.

For more information, see http://seed.ucsd.edu/ ${ }^{\sim}$ freund/NewHomePage/ Applications/Biomedical_Imaging.html 